\title{
THE CENTRAL SUPPORT OF THE PLANCHEREL MEASURE OF AN AFFINE HECKE ALGEBRA
}

\author{
ERIC OPDAM \\ Dedicated to Victor Ginzburg at the occasion of his fiftieth birthday

\begin{abstract}
We give conceptual proofs of certain basic properties of the arrangement of shifted root hyperplanes associated to a root system $R_{0}$ and a $W_{0}=W\left(R_{0}\right)$-invariant real valued parameter function on $R_{0}$. The method is based on the role of this shifted root hyperplane arrangement for the harmonic analysis of affine Hecke algebras. In addition this yields a conceptual proof of the description of the central support of the Plancherel measure of an affine Hecke algebra given in [8].
\end{abstract}

Date: November 23, 2018.

2000 Mathematics Subject Classification. Primary 20C08; Secondary 22D25, 22E35, 43A30.

Key words and phrases. Affine Hecke algebra, Plancherel measure, positivity, residue calculus, support.

During the preparation of this paper the author was partially supported by a Pionier grant of the Netherlands Organization for Scientific Research (NWO). 


\section{Contents}

1. Introduction 2

2. Preliminaries on affine Hecke algebras 4

3. Residue calculus 6

4. Non-cancellation results 11

5. Application of positivity $\quad 13$

6. The support of $\nu$ 18

7. Implications for root systems 20

8. Temperedness of local traces $\chi_{t} \quad 23$

References $\quad 24$

November 23, 2018

\section{Introduction}

Let $V$ be a Euclidean vector space and let $R_{0} \subset V^{*}$ denote a reduced, integral root system with Weyl group $W_{0}$ (we do not assume that $R_{0}$ spans $\left.V^{*}\right)$. Let $k: R_{0} \rightarrow \mathbb{R}$ be a $W_{0}$-invariant real function on $R_{0}$. All that follows depends on this function, but we usually suppress it from the notation.

Theorem 1.1. Let $o=o\left(R_{0}, V, k ; \cdot\right): V \rightarrow \mathbb{Z}$ be the function on $V$ defined by

(1.1) $o(v):=\left|\left\{\alpha \in R_{0} \mid \alpha(v)=k_{\alpha}\right\}\right|-\left|\left\{\alpha \in R_{0} \mid \alpha(v)=0\right\}\right|-\operatorname{dim}(V)$

Then $o \leq 0$, and $o(v)=0$ for at most finitely many $v \in V$.

Observe that Theorem 1.1 implies that the set of zeroes of $o$ is nonempty only if $R_{0}$ spans $V^{*}$.

Theorem 1.2. Let $v \in V$ be such that $o(v)=0$. Then $-v \in W_{0} v$.

The zeroes of $o$ in $V$ are called "residual points" (for $R_{0}$ with respect to the parameter function $k$ ). More generally let $L \subset V$ be an affine subspace. We define

$$
o_{L}:=\left|\left\{\alpha \in R_{0}|\alpha|_{L}=k_{\alpha}\right\}\right|-\left|\left\{\alpha \in R_{0}|\alpha|_{L}=0\right\}\right|-\operatorname{codim}(L)
$$

Let us write $L=v_{L}+V^{L}$ where $V^{L} \subset V$ is a linear subspace and where $\left\{v_{L}\right\}=V_{L} \cap L$ with $V_{L}=\left(V^{L}\right)^{\perp}$. Denote by $R_{L} \subset R_{0}$ the parabolic subsystem of roots that are constant on $L$. Then it is clear that $R_{L} \subset V_{L}^{*}$ and that $o_{L}=o\left(R_{L}, V_{L},\left.k\right|_{R_{L}} ; v_{L}\right)$. Hence Theorem 1.1 implies that $o_{L} \leq 0$ and that $o_{L}=0$ iff $v_{L} \in V_{L}$ is an $R_{L}$-residual point. 
Hence $V_{L}^{*}$ is spanned by $R_{L}$ if $o_{L}=0$. Thus Theorem 1.1 implies that there exist only finitely many affine subspaces $L$ with $o_{L}=0$.

Affine subspaces $L \subset V$ with $o_{L}=0$ are called "residual affine subspaces", and the point $v_{L} \in L$ such that $\left\{v_{L}\right\}=V_{L} \cap L$ is called the center of $L$. The $W_{0}$-orbits of the (centers of the) affine residual subspaces should be thought of as parameter deformations of weighted Dynkin diagrams of nilpotent orbits. The residual affine subspaces or rather their toric analogs called "residual cosets" play a mayor role in the description of the support of the Plancherel measure of the affine Hecke algebra, see Theorem 6.1.

Theorem 1.3. If $L \subset V$ is a residual affine subspace of positive dimension with center $v_{L}$. Then $v_{L}$ is not a residual point for $R_{0}$.

These theorems are not new but can be deduced by combining results of [4] and the appendix A of [8]. This yields proofs based on an elaborate classification of all residual subspaces for all irreducible root systems $R_{0}$ and parameter functions $k$, and then a case-by-case verification of the required properties. In the special case with "equal parameters" (i.e. $k$ is a constant function) there are conceptual proofs available for Theorem 1.1 and Theorem 1.3 (but not for Theorem 1.2 as far as I am aware) based on the interpretation of residual cosets in relation to nilpotent orbits of complex semisimple Lie algebras. More precisely in this special case Theorem 1.1 is a consequence of the Richardson Dense Orbit Theorem [2, Theorem 5.2.1] (see Remark 7.3) whereas Theorem 1.3 follows from the Bala-Carter Theorem [2, Theorem 5.9.5] (see Remark 7.5). In the general unequal parameter case the only proofs that were known to me were based on the complete classification of the residual affine subspaces (see [4], 8]).

The Plancherel formula for affine Hecke algebras was derived in [8] as an application of an elementary uniqueness result for certain multivariable residue distributions applied to an integral representation of the natural trace functional $\tau$ of the Hecke algebra. The method gives complete information on the central support of the Plancherel measure (i.e. the projection of the support to the spectrum of the center) of the affine Hecke algebra and important partial information on the formal dimensions of the discrete series in terms of the central character (a product formula). Yet the situation was unsatisfactory since the Theorems 1.1, 1.2 and 1.3 (lacking general conceptual proofs) were necessary ingredients in order to apply the residue lemma to the harmonic analysis (see [8]). 
In the present paper we remedy the situation and show that the residue method of [8] can be made to work without using the Theorems 1.1, 1.2 and 1.3 as input. We will derive the central support of the Plancherel measure of the Hecke algebra directly from the residue method (see Theorem 6.1), which is the main result of this paper. In addition the Theorems 1.1, 1.2 and 1.3 will follow from this as consequences of the positivity and finiteness of the trace $\tau$ of the affine Hecke algebra (see Section 7 ). We will thus achieve conceptual proofs from basic principles for all these results.

Remark 1.4. These theorems are known to hold for non-integral root systems as well (see [4]). It seems likely that the methods of the present paper also apply in this more general context using degenerate affine Hecke algebras (as in [4]).

It is a pleasure to thank Joseph Bernstein and David Kazhdan for their support and help. Their insightful questions and comments on the "residue method" of [8] made me aware of the possibility of results of this kind.

\section{Preliminaries on affine Hecke algebras}

This section is a quick review of affine Hecke algebras. We refer the reader to [7] or [8] for details and unexplained notations.

Let $W=W_{0} \ltimes X$ be an extended affine Weyl group with translation lattice $X$ and let $S \subset W$ be a set of simple affine reflections. We work with the affine Hecke algebra $\mathcal{H}$ over $\mathbb{C}$ with basis $\left(N_{w}\right)_{w \in W}$ where $N_{s}$ (with $s \in S$ ) satisfies the quadratic relations

$$
\left(N_{s}-q(s)^{1 / 2}\right)\left(N_{s}+q(s)^{-1 / 2}\right)=0
$$

Here $q: S \rightarrow \mathbb{R}_{+}$is positive real and constant on conjugacy classes of simple reflections. We can uniquely extend $q$ to a length multiplicative function $q: W \rightarrow \mathbb{R}_{+}$such that $q(\omega)=1$ for all elements $\omega \in W$ of length 0 . We often write $N_{e}=1$.

2.0.1. Commutative subalgebra $\mathcal{A}$. We define $\mathcal{A} \subset \mathcal{H}$ to be the commutative subalgebra of $\mathcal{H}$ spanned by the Bernstein basis elements $\theta_{x}$ $(x \in X)$. We define $T=\operatorname{MaxSpec}(\mathcal{A})$, a complex torus. We denote by $T_{v}$ the vector group corresponding to $T$, and by $T_{u}$ the compact form of $T$. We have the polar decomposition $T=T_{v} T_{u}$. We assume given a $W_{0}$-invariant inner product on $\operatorname{Lie}\left(T_{v}\right)$ which is rational on $Y$ (the lattice dual to $X)$. We thus consider $\operatorname{Lie}\left(T_{v}\right)$ as a Euclidean space in which $W$ acts as an extended affine Weyl group. 
2.0.2. Center $\mathcal{Z}$. The center $\mathcal{Z} \subset \mathcal{A}$ of $\mathcal{H}$ is given by Bernstein's description $\mathcal{Z}=\mathcal{A}^{W_{0}}$.

2.0.3. Star algebra. The anti-linear anti-involution $*$ of $\mathcal{H}$ given by $N_{w}^{*}=N_{w^{-1}}$ turns $\mathcal{H}$ into a star-algebra.

Observe that $\mathcal{Z} \subset \mathcal{A} \subset \mathcal{H}$, where $\mathcal{Z}$ is a star subalgebra but $\mathcal{A}$ is not (in general).

2.0.4. positivity of trace. We use the standard trace $\tau$ on $\mathcal{H}$ given by $\tau\left(N_{w}\right)=\delta_{w, e}$.

Proposition 2.1. The trace $\tau$ is positive definite with respect to $*$, and $N_{w}$ is an orthonormal basis for the inner product $(x, y):=\tau\left(x^{*} y\right)$.

2.0.5. Eisenstein series. Let $R_{0} \subset X$ be the finite root system attached to $\mathcal{H}$ as usual, i.e. the affine root system of the Coxeter subgroup of $W$ generated by the simple affine reflections is the affine extension of $R_{0}^{\vee}$. We let $\Delta \in \mathbb{C}[T]$ denote the Weyl denominator

$$
\Delta(t)=\prod_{\alpha \in R_{0,+}}\left(1-\alpha(t)^{-1}\right)
$$

We denote by $w_{0}$ the longest element of $W_{0}$.

Proposition 2.2. [7, Proposition 2.24] There exists a unique holomorphic function $E: T \rightarrow \mathcal{H}^{*}\left(\mathcal{H}^{*}\right.$ is the linear dual of $\left.\mathcal{H}\right)$ such that

(i) $E_{t}(1)=\Delta(t)$.

(ii) For all $t \in T, h \in \mathcal{H}, x \in X: E_{t}\left(\theta_{x} h\right)=E_{t}\left(h \theta_{x}\right)=x(t) E_{t}(h)$

2.0.6. Integral formula for trace. We have the following theorem:

Theorem 2.3. [7, Theorem 3.7] Let $p \in T_{v}$ be far in the negative chamber. For all $h \in \mathcal{H}$ :

$$
\tau(h)=\int_{t \in p T_{u}} E_{t}(h) \Delta(t)^{-1} \eta(t)
$$

where

$$
\eta(t)=q\left(w_{0}\right)^{-1} \frac{\Delta(t) \Delta\left(t^{-1}\right)}{D(t) D\left(t^{-1}\right)} d t
$$

with

$$
D(t)=\prod_{\alpha \in R_{0,+}}\left(1-q_{\alpha^{\vee}}^{-1} \alpha(t)^{-1}\right)
$$


Remark 2.4. The statement " $p \in T_{v}$ far in the negative chamber" means that $p$ is sufficiently far from all the walls so that there are no pole hyperplanes of $\eta$ between $p$ and infinity in the negative chamber.

This implies that we can make the distance of $p$ to any wall arbitrarily large without crossing poles (thus without changing the value of the integral).

Remark 2.5. In the case $W=W\left(C_{n}^{\vee}\right)^{a}$, the affine extension of $C_{n}$, the affine Hecke algebra actually allows 3 parameters $q_{i}$. The formulae for $\eta$ are slightly more complicated in this 3 parameter case, but essentially the same. We ignore this case here to keep notations simple, but we remark that all arguments apply to this case equally well.

Corollary 2.6. On the commutative subalgebra $\mathcal{A}=\mathbb{C}[T]$ the trace integral formula simplifies to (with $a \in \mathcal{A}$, and $p \in T_{v}$ far in negative chamber):

$$
\tau(a)=\int_{t \in p T_{u}} a(t) \eta(t)
$$

Remark 2.7. The trace $\tau$ on $\mathcal{A}$ will be studied below. Important ingredients that will play a role are the product structure of $\eta$, the $W_{0}$ invariance of $\eta$, and the elementary Proposition below.

Proposition 2.8. We view $T_{v}$ as a real vector space, and we consider the hyperplane arrangement $\mathcal{P}$ in $T_{v}$ formed by the intersections of the pole hyperplanes of $\eta$ with $T_{v}$. For each central real subarrangement $\mathcal{P}_{c} \subset \mathcal{P}$ of this hyperplane arrangement of poles in $T_{v}$ and for each chamber $C$ of $\mathcal{P}_{c}$, there exists a $w \in W_{0}$ such that $w p \in C$.

Proof. This is obvious: $\mathcal{P}_{c}$ consists of a collection of shifted root hyperplanes of the form $\alpha(t)=q_{\alpha}$ (with $\alpha \in R_{0}$ ) in $T_{v}$ which have a point in common. Hence $C$ is a shift of a union $C^{\prime}$ of Weyl chambers of $W_{0}$. Since $p$ is far in the negative chamber we can choose its distance to any wall much larger than the shifts. In this situation clearly $w p \in C$ iff $w p \in C^{\prime}$. But $W_{0}$ acts transitively on its Weyl chambers, so that there indeed exists a $w \in W_{0}$ such that $w p \in C^{\prime}$.

\section{Residue calculus}

Let us now more generally look at a linear functional $F_{\omega, p}$ on $\mathcal{A}=$ $\mathbb{C}[T]$ defined by

$$
F_{\omega, p}(a)=\int_{p T_{u}} a(t) \omega(t)
$$


where $\omega(t)=P(t) / Q(t) d t$ with $P(t), Q(t)$ products of the form $P(t)=$ $\prod_{m^{\prime} \in \mathcal{M}^{\prime}}\left(1-d_{m^{\prime}}^{-1} x_{m^{\prime}}(t)\right)$ and $Q(t)=\prod_{m \in \mathcal{M}}\left(1-d_{m}^{-1} x_{m}(t)\right)$. Here $\mathcal{M}, \mathcal{M}^{\prime}$ are finite index sets on which we have defined functions $\mathcal{M} \ni m \rightarrow$ $\left(d_{m}, x_{m}\right) \in \mathbb{C}^{\times} \times X$ and $\mathcal{M}^{\prime} \ni m^{\prime} \rightarrow\left(d_{m^{\prime}}, x_{m^{\prime}}\right) \in \mathbb{C}^{\times} \times X$.

Here $p \in T_{v}$ is such that $p T_{u}$ does not meet any of the pole hyperplanes of $\omega$. In other words, if we project each of the pole hyperplanes of $\omega$ to $T_{v}$ along $T_{u}$ then $p$ is a regular element with respect to this affine hyperplane arrangement in $T_{v}$.

Let us define for $m \in \mathcal{M}$ the complex codimension 1 submanifold $L_{m} \subset T$ by

$$
L_{m}=\left\{t \in T \mid x_{m}(t)=d_{m}\right\}
$$

and similarly we define $L_{m^{\prime}}$ for $m^{\prime} \in \mathcal{M}^{\prime}$. We write

$$
T^{m}=\left\{t \in T \mid x_{m}(t)=1\right\}
$$

then $L_{m}$ is a coset of the subgroup $T^{m} \subset T$. Similar notations are used for $\mathcal{M}^{\prime}$

If $L \subset T$ define is any subset then we define

$$
\mathcal{M}_{L}:=\left\{m \in \mathcal{M} \mid L \subset L_{m}\right\}
$$

and similarly we define $\mathcal{M}_{L}^{\prime}$. Using this we define the order $i_{L}=i_{\omega, L}$ of $\omega$ along $L$ by

$$
i_{L}:=\left|\mathcal{M}_{L}\right|-\left|\mathcal{M}_{L}^{\prime}\right|
$$

Definition 3.1. An $\omega$-residual coset $L$ of $T$ is a connected component of the intersection of a subcollection of hypersurfaces $L_{m}$ for $m$ running in a subset of $\mathcal{M}$ (observe that such a component is a coset of a subtorus of $T$ ) which in addition satisfies the property

$$
o_{L}:=i_{L}-\operatorname{codim}(L) \geq 0
$$

The collection of $\omega$-residual cosets is denoted by $\mathcal{L}=\mathcal{L}_{\omega}$.

Definition 3.2. Let $L \in \mathcal{L}$. We define its tempered form $L^{t}$ as follows. We can write $L=b T^{L}$ for some base point $b$ and subtorus $T^{L} \subset T$. Let $T_{L} \subset T$ denote the subtorus whose Lie algebra is orthogonal to $\operatorname{Lie}\left(T^{L}\right)$ (here we use the Euclidean structure on $\operatorname{Lie}\left(T_{v}\right)$, and the fact that $X$ is rational with respect to this structure). Then $L^{t}:=\left(L \cap T_{L}\right) T_{u}^{L}$, which is a compact real form of $L$. The set $\left(L \cap T_{L}\right)$ is finite; we fix an element $r_{L} \in L \cap T_{L}$. The projection of $L^{t}$ onto $T_{v}$ along $T_{u}$ is a point $c_{L} \in T_{v}$, the center of $L^{t}\left(c_{L}\right.$ is the vector part of $\left.r_{L}\right)$.

Remark 3.3. By definition $\mathcal{L}$ is finite. Let $\mathcal{C}$ be the finite set the centers of the elements $L \in \mathcal{L}$.

With these definitions we can now formulate the basic residue lemma: 
Lemma 3.4. There exists a unique collection $\left\{\mathfrak{X}_{c} \in C^{-\infty}\left(c T_{u}\right)\right\}_{c \in \mathcal{C}}$ of distributions such that

(i) The support of $\mathfrak{X}_{c}$ is contained in

$$
S_{c}=\cup_{L \in \mathcal{L}: c_{L}=c} L^{t} \subset c T_{u}
$$

(ii) For all $a \in \mathcal{A}$ :

$$
F_{\omega, p}(a)=\sum_{c \in \mathcal{C}} \mathfrak{X}_{c}\left(\left.a\right|_{c T_{u}}\right)
$$

Remark 3.5. Of course the collection $\left\{\mathfrak{X}_{c}\right\}$ will depend on the chamber $C$ which contains $p$ of the real affine hyperplane arrangement $\mathcal{P}$ in $T_{v}$ formed by the real projections $L_{m} T_{u} \cap T_{v}$ of the pole hyperplanes. If we want to stress this we will write $\mathfrak{X}_{p, c}$.

3.0.7. Local cycles. We can be more precise about the nature of the distributions $\mathfrak{X}_{c}(c \in \mathcal{C})$ by analyzing the existence proof of Lemma 3.4. The result is a description of $\mathfrak{X}_{c}$ as a summation over the $L \in \mathcal{L}$ with $c_{L}=c$ and (for each $L$ ) a sum of normal derivatives of boundary values on $L^{t}$ of certain rational functions on $L$ from certain wedges with edge $L^{t}$. This was done in [8, Proposition 3.7]. Let us describe the result (as we will need it further on):

Let $L \in \mathcal{L}$. We define

$$
\mathcal{M}^{L}=\left\{m \in \mathcal{M}-\mathcal{M}_{L} \mid L \cap L_{m} \neq \emptyset\right\}
$$

and

$$
\mathcal{M}^{L, t}=\left\{m \in \mathcal{M}-\mathcal{M}_{L} \mid L^{t} \cap L_{m} \neq \emptyset\right\} \subset \mathcal{M}^{L}
$$

The set $\mathcal{M}^{L}$ describes the intersection of poles of $\omega$ with $L$, and $M^{L, t}$ the poles of $\omega$ which meet $L^{t}$.

For $\delta>0$ and each $L$ which is a connected component of an intersection of codimension 1 cosets $L_{m} \subset T$ with $m \in \mathcal{M}$, we denote by $\mathcal{B}_{L}\left(r_{L}, \delta\right)$ a ball in $T_{L}$ with radius $\delta$ and center $r_{L}$, and by $\mathcal{B}_{v}^{L}(\delta)$ a ball with radius $\delta$ and center $e$ in $T_{v}^{L}$.

Let $U^{L}(\delta) \subset T^{L}$ be the open set $\left\{t \in T^{L} \mid \forall m \in \mathcal{M}^{L}: t \overline{\mathcal{B}_{L}\left(r_{L}, \delta\right)} \cap\right.$ $\left.L_{m}=\emptyset\right\}$. Note that $U^{L}\left(\delta_{1}\right) \subset U^{L}\left(\delta_{2}\right)$ if $\delta_{1}>\delta_{2}$, and that the union of these open sets is equal to the complement of union of the codimension 1 subsets $r_{L}^{-1}\left(L \cap L_{m}\right) \subset T^{L}$ with $m \in \mathcal{M}^{L}$.

Proposition 3.6. Let $\epsilon>0$ be such that for all $m \in \mathcal{M}$ and $L \in \mathcal{L}$, $L_{m} \cap \mathcal{B}_{L}\left(r_{L}, \epsilon\right) \mathcal{B}_{v}^{L}(\epsilon) T_{u}^{L} \neq \emptyset$ implies that $L^{t} \cap L_{m} \neq \emptyset$. There exist

(i) $\forall L \in \mathcal{L}$, a point $\epsilon^{L} \in \mathcal{B}_{v}^{L}(\epsilon) \backslash \cup_{m \in \mathcal{M}^{L, t}} T^{m}$,

(ii) $a 0<\delta<\epsilon$ such that $\forall L \in \mathcal{L}^{\omega}, \epsilon^{L} T_{u}^{L} \subset U^{L}(\delta)$, and 
(iii) $\forall L \in \mathcal{L}$, a compact cycle $\xi_{L} \subset \mathcal{B}_{L}\left(r_{L}, \delta\right) \backslash \cup_{m \in \mathcal{M}_{L}} L_{m}$ of $d i$ mension $\operatorname{dim}_{\mathbb{C}}\left(T_{L}\right)$,

such that $\forall c \in \mathcal{C}^{\omega}, \forall \phi \in C^{\infty}\left(c T_{u}\right)$ :

$$
\mathfrak{X}_{c}(\phi)=\sum_{\left\{L \mid c_{L}=c\right\}} \mathfrak{X}_{L}(\phi),
$$

where $\mathfrak{X}_{L}$ is the distribution on $c T_{u}$ with support $L^{t}$ defined by $\forall a \in \mathcal{A}$ :

$$
\mathfrak{X}_{L}(a)=\int_{\epsilon^{L} T_{u}^{L} \times \xi_{L}} a \omega .
$$

If $\mathcal{M}^{L, t}=\emptyset$ we may take $\epsilon^{L}=e$.

Remark 3.7. We remark that the splitting of $\mathfrak{X}_{c}$ as a sum of $\mathfrak{X}_{L}$ as given here has in general no intrinsic meaning (it is not unique, and the cycles $\xi_{L}$ are not uniquely determined). Of course the ambiguity of $\mathfrak{X}_{L}$ is restricted to distributions with support in the intersection of $L^{t}$ with other tempered residual cosets $M^{t}$.

We remark that (i), (ii) and (iii) imply that the functional $\mathfrak{X}_{L}$ on $\mathcal{A}$ indeed defines a distribution on $c_{L} T_{u}$, supported on $L^{t}$. We look at it more closely in order to arrive at a useful conclusion.

The cycle of integration of $\mathfrak{X}_{L}$ is the product $\epsilon^{L} T_{u}^{L} \times \xi_{L} \subset T^{L} \times T_{L}$. Given $L \in \mathcal{L}$ and $t=t^{L} t_{L} \in T$ we write

$$
\omega(t)=\left(P^{L}\left(t^{L} t_{L}\right) / Q^{L}\left(t^{L} t_{L}\right)\right)\left(P_{L}\left(t_{L}\right) / Q_{L}\left(t_{L}\right)\right) d t_{L} \wedge d t^{L}
$$

with $P_{L}$ and $Q_{L}$ the product over the factors parametrized by $\mathcal{M}_{L}^{\prime}$ and $\mathcal{M}_{L}$ respectively, and where $P^{L}$ and $Q^{L}$ are the products of the remaining factors. Here we have chosen the orientations for $T_{u}^{L}$ and $T_{L, u}$ such that if $d t^{L}$ and $d t_{L}$ denote the holomorphic continuations of the normalized volume forms of $T_{u}^{L}$ and $T_{L, u}$ then $d t=d t_{L} \wedge d t^{L}$.

Hence we may write

$$
\mathfrak{X}_{L}(a)=\int_{\epsilon^{L} T_{u}^{L}} I\left(a, t^{L}\right) d t^{L}
$$

where $I\left(a, t^{L}\right)$ denotes the inner integral

$$
\left.I\left(a, t^{L}\right):=\int_{t^{L} \xi} a\left(t^{L} t_{L}\right) P^{L}\left(t^{L} t_{L}\right) / Q^{L}\left(t^{L} t_{L}\right)\right)\left(P_{L}\left(t_{L}\right) / Q_{L}\left(t_{L}\right)\right) d t_{L}
$$

Hence

$$
I\left(a, t^{L}\right)=\left.D\left(a P^{L} / Q^{L}\right)\right|_{r_{L} t^{L}}
$$

where $D \in \operatorname{Sym}\left(\operatorname{Lie}\left(T_{L}\right)\right)$ is a constant coefficient differential operator on $T_{L}$, extended to $T$ by invariance, homogeneous of degree

$$
o_{L}:=i_{L}-\operatorname{codim}(L)
$$


Hence $I(a, t)$ is a linear combination of (possibly higher order) partial derivatives $D_{\kappa} a$ of $a$ at $r_{L} t$ in the direction of $T_{L}$, with coefficients in the ring of rational functions on $T^{L}$ which are regular outside the codimension 1 intersections $r_{L}^{-1}\left(L \cap L_{m}\right)$

$$
I(a, t)=\sum_{\kappa} f_{\kappa} D_{\kappa} a .
$$

Indeed, by the Leibniz rule the coefficient $f_{\kappa}$ is the restriction to $L$ of a sum of normal derivatives $D_{\kappa}^{\nu}\left(P^{L} / Q^{L}\right)$ of degree

$$
\operatorname{deg}\left(D_{\kappa}^{\nu}\right)=o_{L}-\operatorname{deg}\left(\mathrm{D}_{\kappa}\right)
$$

Hence $\mathfrak{X}_{L}(a)$ is equal to the sum of the boundary value distributions $\mathrm{BV}_{\epsilon^{L}, f_{\kappa}}$ of the meromorphic coefficient functions, applied to the corresponding partial derivative $D_{\kappa} a$ of $a$, restricted to $L^{t}$ :

$$
\mathfrak{X}_{L}(a)=\sum_{\kappa} \mathrm{BV}_{\epsilon^{L}, f_{\kappa}}\left(\left.D_{\kappa} a\right|_{L^{t}}\right) \text {. }
$$

We see that $\mathfrak{X}_{L}$ is a distribution supported in $L^{t} \subset c_{L} T_{u}$, which only depends on $\xi_{L}$ and on the component of $\mathcal{B}_{v}^{L}(\epsilon) \backslash \cup_{m \in \mathcal{M}^{L, t e m p}} T^{m}$ in which $\epsilon^{L}$ lies.

The following corollary is the main result of this subsection:

Corollary 3.8. Let $L \in \mathcal{L}$. Suppose that $\mathfrak{X}_{L} \neq 0$ (recall that this depends on $p$, and in general on the choices of the $\left.\epsilon^{L}\right)$. Recall that $T$ is finite quotient of $T^{L} \times T_{L}$ by $K_{L}=T_{L} \cap T^{L}$, a finite abelian group. There exists an element $b \in \mathbb{C}\left[T_{L}\right]^{K_{L}} \subset \mathbb{C}[T]$ such that

(i) The vanishing order of $b$ at $r_{L}$ is $o_{L}$.

(ii) For all $a \in \mathbb{C}[T]$ we have $\mathfrak{X}_{L}(a b)=B V_{\epsilon^{L}, \omega^{L}}\left(\left.a\right|_{L^{t}}\right)$, where we mean by $B V_{\epsilon^{L}, \omega^{L}}$ the boundary value distribution on $L^{t}$ defined by

$$
B V_{\epsilon^{L}, \omega^{L}}(f)=\lim _{\epsilon^{L} \rightarrow 1} \int_{t \in \epsilon^{L} T_{u}^{L}} f\left(r_{L} t\right) \omega^{L}\left(r_{L} t\right)
$$

where for $t \in \epsilon^{L} T_{u}^{L}$ we put

$$
\omega^{L}\left(r_{L} t\right):=P^{L}\left(r_{L} t\right) / Q^{L}\left(r_{L} t\right) d^{L} t
$$

Proof. We have

$$
I(a b, t)=\left.D\left(a b P^{L} / Q^{L}\right)\right|_{r_{L} t}
$$

where $D \in \operatorname{Sym}\left(\operatorname{Lie}\left(T_{L}\right)\right)$ is a constant coefficient differential operator on $T_{L}$, extended to $T$ by invariance, nonzero (by assumption) and homogeneous of order $o_{L}$. It is then elementary that we can choose $b$ so 
that

$$
I(a b, t)=a\left(r_{L} t\right) P^{L}\left(r_{L} t\right) / Q^{L}\left(r_{L} t\right)
$$

as required.

Corollary 3.9. Suppose that $F_{\omega, p}$ is a (complex) measure on $S=$ $\cup_{c \in \mathcal{C}} S_{c}$ (in the sense of Lemma 3.4). Suppose moreover that $\mathfrak{X}_{L} \neq 0$ and that $L^{t}$ is not contained in the tempered form $M^{t}$ of a larger residual subspace $M$. Then $o_{L}=0$.

Proof. Let $\phi$ be a smooth function on $c T_{u}$ (with $c$ being the center of $L$ ) whose support is contained in the complement of the union of the tempered residual cosets $M^{t}$ with $M \neq L$ and with center $c$. Using the notion of approximating sequences (cf. [8, Lemma 3.5]) we can construct for any $N \in \mathbb{N}$ a sequence $\left\{a_{n}\right\}$ in $\mathcal{A}$ such that $\left.\left.D\left(a_{n}\right)\right|_{L^{t}} \rightarrow D(\phi)\right|_{L^{t}}$ uniformly for all constant coefficient differential operators $D$ on $T$ of order at most $N$, and $\left.D\left(a_{n}\right)\right|_{M^{t}} \rightarrow 0$ uniformly for all $M \neq L$ (we construct $a_{n}$ as in the proof of [8, Lemma 3.19(ii)]). Suppose that $o_{L}>0$ and let $b \in \mathbb{C}\left[T_{L}\right]$ be as in Corollary 3.8. Then the sequence $a_{n} b$ converges uniformly to 0 on the support of $F_{\omega, p}$ and by the assumption that $F_{\omega, p}$ is a complex measure we conclude that $F_{\omega, p}\left(a_{n} b\right) \rightarrow 0$. Moreover $\mathfrak{X}_{M}\left(a_{n} b\right) \rightarrow 0$ (for $M \neq L$ ) by the properties of the sequence $a_{n}$ and since all $\mathfrak{X}_{M}$ are finite order distributions (choose $N$ sufficiently large). We conclude that $\mathfrak{X}_{L}\left(a_{n} b\right) \rightarrow 0$. On the other hand, Corollary 3.8 implies that

$$
\mathfrak{X}_{L}\left(a_{n} b\right) \rightarrow B V_{\epsilon^{L}, \omega^{L}}(\phi)
$$

and from the assumption that $L^{t}$ is not properly contained in another tempered residual coset $M^{t}$ it is clear that we may choose $\phi$ in such a way that this expression is nonzero. This is the required contradiction.

\section{Non-cancellation results}

Recall the following results of [8]:

Lemma 4.1. ([8, Lemma A.11]) Assume that $\omega$ is such that $o_{L}=0$ for all $L \in \mathcal{L}$ with $\operatorname{dim}(L)>0$. Let $r \in \mathcal{L}$ be a residual point for $\omega$. There exists a $L \in \mathcal{L}^{\omega}$ such that $\operatorname{dim}(L)=1$ and $r \in L$.

Theorem 4.2. ([8, Theorem 3.29]) Suppose that $\omega$ is such that

1. $o_{L}=0$ for all $L \in \mathcal{L}$.

2. If $L, M \in \mathcal{L}$ and $L \neq M$ then $L^{t} \not \subset M^{t}$. 
Let $r \in \mathcal{L}$ be a residual point. Then there exists a chamber $C$ of $T_{v}^{r e g}:=$ $T_{v} \backslash \cup_{m \in \mathcal{M}} T_{v} \cap L_{m} T_{u}$ such that for $p \in C$ we have $\mathfrak{X}_{p, r}(a)=d_{p, r} a(r)$ for some $d_{p, r} \neq 0$.

Proof. This is essentially just [8, Theorem 3.29], although there the result was only formulated for the special case of the integral formula of the trace $\tau$ of $\mathcal{H}$ restricted to $\mathcal{A}$. The arguments apply to the general case as well. Let us discuss the arguments briefly here.

When one computes $\mathfrak{X}_{p, r}$ under the simplifying assumption 2 one chooses a path from $p$ to $e$ in $T_{v}$ which intersects each pole hyperplane $L_{m}$ at most once and at points of the form $r_{L_{m}} t^{m}$ (with $t \in T_{v}^{m}$ and regular for $r_{L_{m}}^{-1}\left(L_{m} \cap L_{l}\right)$ with $\left.l \in M^{L_{m}}\right)$. When we move $p$ along this path to $e$ we see by virtue of assumption 1 that a residue at $r_{L, m} t$ is picked up which is of the form $d_{m} F_{\omega^{m}, T^{m}}$ (notations as in equation (3.22) (with $d_{m}$ a constant) applied to the restriction of $a \in \mathcal{A}$ to $L_{m}=r_{L} T^{m}$ (and then transported to $T^{m}$ via the map $t \rightarrow r_{L_{m}} t$ ). One now continues by moving the regular points $t \in T_{v}^{m}$ to the identity $e$ of $T_{v}^{m}$ along paths in $T^{m}$ as above etc. etc. At the last step of this process one has one-dimensional integrals on the one dimensional tori $T^{L}$ of the form $d_{L} F_{\omega^{L}, t^{L}}$ with $t^{L} \in T_{v}^{L}$ and $d_{L}$ a constant depending on $p$ applied to the restriction of $a \in \mathcal{A}$ to $L=r_{L} T^{L}$. If we want to know $\mathfrak{X}_{p, r}$ we need to move the $t^{L} \in T_{v}^{L}$ to $e \in T_{v}^{L}$ for all the residual lines containing $r$ and see if a residue at $r$ is picked up. Then we add the result, which will in general be a big summation of constants time the delta distribution of $r$.

By the Lemma above it is clear that there exists at least one residual line through $r$. But the problem is cancellation, as we have to add several complex constants $d_{L}$ coming from the residual lines containing $r$ in order to compute $\mathfrak{X}_{p, r}$. We need to show that these constants do not cancel at $r$ for at least one choice of $p$.

The argument in [8, Theorem 3.39] is an induction with respect to the dimension of $T$. Assume by induction that it is true for tori of dimension $n-1$. Then it is shown in the proof of [8, Theorem 3.39] that one can choose $p$ and the paths from $t^{L}$ to $e$ in the residual cosets $L$ of dimension at least 2 in such a way that among the nonzero residues picked up at one of the residual lines $L$ through $r$ (at a point $r_{L} t$ with $\left.t \in T_{v}^{L}\right)$ precisely one of these one-dimensional residual integrals has the property that $r_{v}$ is between $\left(r_{L}\right)_{v}$ and $\left(r_{L}\right)_{v} t$. Here we used that $\left(r_{L}\right)_{v} \neq r_{v}$, as a consequence of assumption 2. Hence in the final step of the residue computations in which we move the base points of the residual integrals in the one dimensional residual cosets $L$ from $\left(r_{L}\right) t$ 
to $r_{L}$, we pick up precisely one nonzero residue at $r$ corresponding to a first order pole of $\omega^{L}$. This implies the result.

The following result is a slight generalization which will be handy for later applications.

Lemma 4.3. Suppose that $\omega$ is such that

1. $o_{L}=0$ for all $L \in \mathcal{L}$ with $\operatorname{dim} L>0$.

2. If $L, M \in \mathcal{L}$ and $L \neq M$ then $L^{t} \not \subset M^{t}$.

Let $r \in \mathcal{L}$ be a residual point with $o(r):=o_{\{r\}}=k \geq 0$. Then there exists a chamber $C$ of $T_{v}^{r e g}:=T_{v} \backslash \cup_{m \in \mathcal{M}} T_{v} \cap L_{m} T_{u}$ such that for $p \in C$ we have $\mathfrak{X}_{p, r}(a)=D a(r)$ for some differential operator $D$ of order $k$ with highest order term of the form $d_{p, r} X^{k}(a)(r)$ where $X$ is a constant vector field on $T$ corresponding to a one parameter subgroup of $T$ in the direction of one of the one dimensional residual cosets through $r$.

Proof. This is similar to the proof that was outlined above, except that in the last stage of the computation of the one-dimensional residues we need to take the residue of $a \omega^{L}$ at $r$ where $\omega^{L}$ has a pole of order $k$ at $r$. This proves the result.

Remark 4.4. Here we really need to assume that $o_{L}=0$ for all the higher dimensional residual cosets. Without this assumption things become more complicated and we do not have a simple non-cancellation result.

\section{Application of positivity}

We return to the situation of paragraph 2.0.6.

Proposition 5.1. The restriction of $\tau$ to $\mathcal{Z}$ defines a Radon probability measure $\nu$ on $W_{0} \backslash T$ whose support (viewed as a $W_{0}$-invariant subset of $T$ ) is contained in the union $S$ of the tempered forms $L^{t}$ where $L$ runs over the $\eta$-residual cosets (note that this is a $W_{0}$-invariant set since $\eta$ is $W_{0}$-invariant). Moreover, if $t \in S$ is in the support of $\tau$ then $t^{*}=\bar{t}^{-1} \in W_{0} t$.

Proof. In [8], paragraph 3.3.2, this was shown with $S$ replaced by the larger collection $S^{\mathrm{qu}}$ of $\eta / \Delta$-residual subspaces (the argument showing that $\nu$ is a probability measure is based on the positivity of $\tau$ on $\mathcal{H}$ ). Observe that $S^{\text {qu }}$ too is a $W_{0}$-invariant collection of cosets (since $\Delta$ is essentially skew invariant). In [8], subsection 3.4 it was remarked that the support of $\left.\tau\right|_{\mathcal{A}}$ is actually contained in $S$. We give a slightly 
different argument here. By Lemma 3.4 applied to the restriction of $\tau$ to $\mathcal{A}$ (Corollary 2.6) we see that $\tau$ defines distributions $\mathfrak{X}_{c} \in C^{-\infty}\left(c T_{u}\right)$ with $c$ running over the centers of $\eta$-residual cosets, with $\mathfrak{X}_{c}$ supported on $S_{c} \subset S_{c}^{\text {qu }}$ (the extra factor $\Delta$ in the denominator cancels on $\mathcal{A}$, this is the point here). The $W_{0}$-average in each $W_{0}$-orbit of centers $W_{0} c \in \mathcal{C}$ defines a $W_{0}$-invariant collection of $W_{0, c}$-invariant distributions $\mathfrak{Y}_{c} \in$ $C^{-\infty}\left(c T_{u}\right)$ supported on $S_{c} \subset S_{c}^{\text {qu }}$. Hence the difference of the sum of this $W_{0}$-invariant collection of distributions and $\nu$, which is the sum of $W_{0}$-invariant collection of positive measures $\nu_{c}$ supported on $S_{c}^{\mathrm{qu}}$ (also $W_{0, c}$-invariant by definition) defines the 0 -functional on $\mathcal{A}$. By the uniqueness assertion of the residue lemma 3.4 we have the equality $\nu_{c}=\mathfrak{Y}_{c}$ for each $\eta / \Delta$-residual center $c \in \mathcal{C}^{\mathrm{qu}}$, implying the result.

Remark 5.2. We stress that the proofs of these facts use no special properties other than the $W_{0}$-invariance of $\eta$, the positivity of $\tau$ on $\mathcal{H}$, and the fact that the integral formula for $\tau$ on $\mathcal{A}$ simplifies by cancellation of a factor $\Delta$. In particular we have not used knowledge obtained by the classification of residual cosets.

Remark 5.3. As in the proof above we will write $\nu_{c}$ for the $W_{0, c^{-}}$ invariant measure on $c T_{u}$ (with $c \in \mathcal{C}$ ) defined by restriction of $\nu$.

Another important remark which follows from positivity is the following. For any $h \in \mathcal{H}$ we define a collection "local distributions" $\mathfrak{X}_{c}^{h} \in C^{-\infty}\left(c T_{u}\right)$ where $c$ runs over the set $\mathcal{C}^{\text {qu }}$ of centers of $\eta / \Delta$-residual cosets by applying the residue lemma 3.4 to the integral formula for the functional $\mathcal{A} \ni a \rightarrow \tau(h a)$ on $\mathcal{A}$ (as a special case we have $\mathfrak{X}_{c}=\mathfrak{X}_{c}^{1}$ ). Again following [8] we symmetrize these local distributions over $W_{0}$ (as in the proof of Proposition 5.1) in order to obtain a $W_{0}$-invariant collection of distributions $\mathfrak{Y}_{c}^{h} \in C^{-\infty}\left(c T_{u}\right)^{W_{0, c}}$ which is uniquely characterized by

$$
\tau(z h)=\sum_{c \in W_{0} \backslash \mathcal{C}^{\mathrm{qu}}} \mathfrak{Y}_{c}^{h}\left(\left.z\right|_{c T_{u}}\right)
$$

for all $z \in \mathcal{Z}$.

By application of the positivity of the trace and the basic fact that for any hermitian element $h$ of $\mathcal{H}$ the element $h+\|h\|_{o} 1 \in \mathcal{H}_{+}$is a positive element, one can prove the following fundamental result:

Theorem 5.4. [8, Corollary 3.23] For all $h \in \mathcal{H}$ and all $c \in \mathcal{C}^{\mathrm{qu}}$ $\mathfrak{Y}_{c}^{h}$ is absolutely continuous with respect to $\nu_{c}$. In fact we have for all continuous $W_{0, c}$-invariant functions $\phi$ on $c T_{u}$ that

$$
\left|\mathfrak{Y}_{c}^{h}(\phi)\right| \leq\|h\|_{o} \nu_{c}(\phi)
$$


where $\|h\|_{o}$ denotes the norm of $h$ in the left regular representation (with respect to the Hilbert norm on $\mathcal{H}$ defined by the $\tau$ and $*$ as in the first section).

Corollary 5.5. For all $h \in \mathcal{H}$ the symmetrized local distributions $\mathfrak{Y}_{c}$ are supported on $S$ (as opposed to the larger set $S^{\mathrm{qu}}$ ).

Definition 5.6. (see [8, Corollary 3.23]) In particular, there exists a unique $\nu$-integrable $W_{0}$-invariant function $t \rightarrow \chi_{t} \in \mathcal{H}^{*}$ on $T$, defined on the support of $\nu$, which is for $\nu$-almost every $t \in S$ a positive tracial state on $\mathcal{H}$ with central character $W_{0} t$ (i.e. it is a positive trace, $\chi_{t}(1)=$ 1 , and $\chi_{t}(z h)=z(t) \chi_{t}(h)$ for all $\left.t \in S\right)$ such that for all $h \in \mathcal{H}$ :

$$
\tau(h)=\int_{S} \chi_{t}(h) d \nu(t)
$$

Corollary 5.7. The support of $\nu$ on $W_{0} \backslash T$ is equal to the central support of the Plancherel measure of $\mathcal{H}$.

Proof. The local traces $\chi_{t}$ for $t \in \operatorname{Supp}(\nu)=S$ can be decomposed as a positive finite linear combination of irreducible characters of $\mathcal{H}$, each having central character $W_{0} t$. This further refinement of the decomposition (5.3) is the Plancherel formula of $\mathcal{H}$, and the (closure of) the set of irreducible characters $\mathcal{H}$ that arises in this way is the support of the Plancherel measure. Hence the support of $\nu$ is the projection of the support of the Plancherel measure to $\operatorname{Max} \operatorname{Spec}(\mathcal{Z})=W_{0} \backslash T$.

Corollary 5.8. The local distributions $\mathfrak{X}_{c}$ arising from the application of Lemma 3.4 to the restriction of $\tau$ to $\mathcal{A}$ are themselves positive Radon measures supported on $S$ such that $\mathfrak{X}=\sum_{c \in \mathcal{C}} \mathfrak{X}_{c}$ is a (nonsymmetric) probability measure on $T$.

Proof. Fix $c \in \mathcal{C}$. We have by definition for all $a \in \mathcal{A}$ and $\phi \in$ $C^{\infty}\left(c T_{u}\right)^{W_{0, c}}$ :

$$
\mathfrak{Y}_{c}^{a}(\phi)=\left|W_{0} / W_{0, c}\right| \int_{S_{c}} \phi(t) \chi_{t}(a) d \nu(t)
$$

Since $\chi_{t}(\cdot)$ is a positive tracial state for $\nu$-almost all $t \in S_{c}$ which has central character $W_{0} t$, the GNS construction implies that $\chi_{t}$ is $\nu$ almost everywhere a positive real linear combination of irreducible (and thus finite dimensional) unitary characters of $\mathcal{H}$ with central character $W_{0} t$. It follows (using the finite dimensionality of the irreducibles) that there exists an essentially unique (i.e. modulo bounded real functions supported on a $\nu$-null set) real non-negative function $d: S_{c} \rightarrow \mathbb{R}_{\geq 0}$ 
such that

$$
\chi_{t}(a)=\sum_{s \in W_{0} t} d(s) a(s)
$$

By this formula it is clear that we can extend the application $\mathcal{A} \ni a \rightarrow$ $\chi_{t}(a)$ for fixed $t \in S_{c}$ continuously to an application $C^{\infty}\left(c T_{u}\right) \ni \phi \rightarrow$ $\chi_{t}(\phi)$ and with this definition we have for all $t \in S_{c}$ that

$$
\chi_{t}(\phi)=\sum_{s \in W_{0} t} d(s) \phi(s)
$$

On the other hand, by Lemma 5.9 there are for each $\phi \in C^{\infty}\left(c T_{u}\right)$ finitely many $a_{i} \in \mathcal{A}$ and $\phi_{i} \in C^{\infty}\left(c T_{u}\right)^{W_{0, c}}$ such that $\phi=\sum a_{i} \phi_{i}$ and thus (5.5) and (5.6) yield

$$
\chi_{t}(\phi)=\sum_{i} \phi_{i}(t) \chi_{t}\left(a_{i}\right)
$$

This shows that for all $\phi \in C^{\infty}\left(c T_{u}\right)$ the function $t \rightarrow \chi_{t}(\phi)$ is $\nu$ integrable.

There exists a stratification of $c T_{u}$ by finitely many locally closed subsets $D_{i}$ such that for all $x \in D_{i}$ there exists an open set $U_{x} \subset D_{i}$ with the property that for all $y \in U_{x}$ we have $W_{0} y \cap U_{x}=y$.

For every $x \in D_{i} \cap S_{c}$ we can find a $\phi \in C^{\infty}\left(c T_{u}\right)$ which is supported on a small neighborhood $U$ of $x$ such that $U_{x}=U \cap D_{i}$ is as above. By (5.6) we see that for such choice of $\phi$ we have for all $s \in U_{x}$ :

$$
d(s) \phi(s)=\chi_{s}(\phi)
$$

It follows that the restriction of $d(s)$ to each $D_{i}$, and hence $d(s)$, is integrable.

In view of (5.4), (5.5) and (5.6) this implies that the application

$$
\mathcal{A} \ni a \rightarrow \mathfrak{Y}_{c}^{a}(1)=\left|W_{0} / W_{0, c}\right| \int_{S_{c}} \sum_{s \in W_{0} t} d(s) a(s) \nu(t)
$$

extends to a positive measure on $c T_{u}$ with support contained in $W_{0} S_{c}$. By definition these measures have the property (for all $a \in \mathcal{A}$ ) that

$$
\tau(a)=\sum_{c \in \mathcal{C}} \mathfrak{Y}_{c}^{a}(1)
$$

and thus, again by the uniqueness assertion of Lemma 3.4, it follows that

$$
\mathfrak{X}_{c}(a)=\mathfrak{Y}_{c}^{a}(1)
$$

and thus that $\mathfrak{X}_{c}$ is indeed a positive measure. 
Lemma 5.9. The $C^{\infty}\left(c T_{u}\right)^{W_{0, c}}$-module $C^{\infty}\left(c T_{u}\right)$ can be generated by a finite set $E \subset \mathbb{C}\left[c T_{u}\right]$.

Proof. Here we identify $c T_{u}$ with the compact torus $T_{u}$ via $c t \rightarrow t$ so that we can speak of the space $\mathbb{C}\left[c T_{u}\right]$ of Laurent polynomials on $c T_{u}$. We identify $\mathcal{A}$ with $\mathbb{C}\left[c T_{u}\right]$ via restriction of $a \in \mathcal{A}$ to $c T_{u} \subset T$.

We have a smooth action of a finite group $G=W_{0, c}$ on this compact torus which comes from a linear action of $G$ on the character lattice $X$. It is then easy to see that there exists a real vector space $V$ with an orthogonal linear action of $G$ on $V$ and a $G$-equivariant proper embedding $i: c T_{u} \rightarrow V$ such that $\mathbb{C}\left[c T_{u}\right]=i^{*}(\mathbb{C}[V])$ and $\mathbb{C}\left[c T_{u}\right]^{G}=$ $i^{*}\left(\mathbb{C}[V]^{G}\right)$. We have $C^{\infty}\left(c T_{u}\right)=i^{*}\left(C^{\infty}(V)\right)$, and we can also easily see that (see e.g. [6, paragraph 5]) $C^{\infty}\left(c T_{u}\right)^{G}=i^{*}\left(C^{\infty}(V)^{G}\right)$.

Since $G$ is finite, $\mathbb{C}[V]$ is a finitely generated $\mathbb{C}[V]^{G}$-module. Indeed, we have the standard $\mathbb{C}[V]^{G}$-module isomorphism

$$
\begin{aligned}
& \bigoplus_{\pi \in \operatorname{Irr}(G)} \mathbb{C}\left[V, V_{\pi}^{*}\right] \otimes V_{\pi} \rightarrow \mathbb{C}[V] \\
& p \otimes v \rightarrow\langle p, v\rangle
\end{aligned}
$$

and the $\mathbb{C}[V]^{G}$-modules of equivariant polynomials $\mathbb{C}\left[V, V_{\pi}^{*}\right]$ are known to be finitely generated. Let $F_{\pi} \subset \mathbb{C}\left[V, V_{\pi}^{*}\right]$ be a finite set of generators. By a result of Poénaru [9, p.106], it is known that $F_{\pi}$ also generates the space of equivariant smooth functions $C^{\infty}\left(V, V_{\pi}^{*}\right)$ as a module over $C^{\infty}(V)^{G}$.

Hence similar to the argument above, $C^{\infty}(V)$ is generated over $C^{\infty}(V)^{G}$ by the union $E_{V} \subset \mathbb{C}[V]$ of the sets $E_{\pi}$ consisting of the elements of the form $\left\langle f, v_{i}\right\rangle$ with $f \in F_{\pi}$ and $v_{i}$ running over a basis of $V_{\pi}$.

Via the surjective algebra homomorphism $i^{*}$ we see that $E=i^{*}\left(E_{V}\right) \subset$ $\mathbb{C}\left[c T_{u}\right]$ is a set of generators of $C^{\infty}\left(c T_{u}\right)$ over $C^{\infty}\left(c T_{u}\right)^{G}$.

Remark 5.10. In general $\mathfrak{X}_{c}^{h}$ can be of higher order as a distribution. What goes wrong in the above argument is the fact that formula 5.5 does not hold for the functional $a \rightarrow \chi_{t}(a h)$ for an arbitrary $h \in \mathcal{H}$. Indeed, the "off-diagonal" matrix elements of $\mathcal{A}$ acting in a unitary irreducible representation $V$ of $\mathcal{H}$ are not in general linear combinations of characters of $\mathcal{A}$ if $\mathcal{A}$ acts non-semisimply. This may be the case since $\mathcal{A}$ is not a $*$-subalgebra. It is of course well known that this phenomenon indeed occurs.

Using the integral formula for $\tau$ in 2.0.6 we see in this way that the length of the indecomposable blocks of the restriction of an irreducible tempered representation $V$ to $\mathcal{A}$ is bounded by the zero order of $\Delta$ at the central character of $V$. 
Remark 5.11. In some sense $\mathfrak{X}$ is a nonsymmetric version of the Plancherel measure of $\mathcal{Z}$ with respect to $\tau$. Its non-symmetry encodes the distribution of the $\mathcal{A}$-weight spaces in the collection of tempered representations carried by a given central character in the support of $\nu$.

\section{The support of $\nu$}

We now put things together in order to prove that the support of the Plancherel measure $\nu$ is equal to $S$. At the same time we derive certain fundamental properties of the configuration of poles and zeroes of $\eta$.

Theorem 6.1. Let $\mathcal{L}$ denote the collection of residual cosets for $\eta$. The following assertions hold true:

(A) For every $\eta$-residual coset $L$ with center $c \in T_{v}$ there exists $a w \in W_{0}$ such that $w L^{t}$ is contained in the support of the measure $\mathfrak{X}_{w c}$.

(B) For all $L \in \mathcal{L}: o_{L}=0$.

(C) For all $M, L \in \mathcal{L}$ with $L \neq M: M^{t} \not \subset L^{t}$.

In particular, the support of $\nu$ (and thus the central support of the Plancherel measure, cf. Corollary 5.7) equals the union of the tempered forms $L^{t}$ of the $\eta$-residual cosets.

Proof. The last assertion follows from (A). Indeed, by (5.11) in the proof of Corollary 5.8 we see that the support of $\mathfrak{X}_{c}$ is equal to the support of $a \rightarrow \mathfrak{Y}_{c}^{a}(1)$, which by (5.9) is contained in the support of $\nu_{c}=\mathfrak{Y}_{c}^{1}$. This is a $W_{0^{-}}$-invariant set, hence we conclude that the $W_{0^{-}}$ orbit of the support of $\mathfrak{X}_{c}$ is contained in the support of $\nu_{c}$. Conversely, since $\nu_{c}=\mathfrak{Y}_{c}^{1}$ is the restriction to $\mathcal{Z}$ of $\mathfrak{X}_{c}$ we see that its support must be contained in the $W_{0}$-orbit of the support of $\mathfrak{X}_{c}$. This proves the assertion.

Now we turn to the proof of (A),(B), and (C). We do this simultaneously with induction to the codimension of the residual cosets. Consider the following assertions:

(A-k) For every $\eta$-residual coset $L$ with center $c \in T_{v}$ and $\operatorname{codim}(L) \leq$ $k$ there exists a $w \in W_{0}$ such that $w L^{t}$ is contained in the support of the measure $\mathfrak{X}_{w c}$.

(B-k) For all $L \in \mathcal{L}$ with $\operatorname{codim}(L) \leq k: o_{L}=0$.

(C-k) For all $M, L \in \mathcal{L}$ with $M \neq L$ and $\operatorname{codim}(L) \leq k: M^{t} \not \subset L^{t}$.

Start of the induction: (B-0) obviously holds. (C-0) holds too, since $T^{t}:=T_{u}$ meets none of the poles $\alpha(t)=q_{\alpha^{\vee}}$ of $\eta$ (indeed, for this to happen one should have $q_{\alpha^{\vee}}=1$. This is allowed, but in that case the 
pole will cancel against a factor of $\Delta(t)$ in de numerator of $\eta$ ). Finally (A-0) holds, in view of Proposition 3.6, and $\mathfrak{X}_{e}(a)$ is simply $\int_{T_{u}} a \eta$. We note that we can take $\epsilon^{T}=1$ here in Proposition 3.6 since (as we remarked in the proof of $(\mathrm{B}-0))$ no pole meets $T_{u}$. In particular, $\mathfrak{X}_{e}$ is a smooth, nonzero measure on $T_{u}$.

Assume(A-l), (B-l) and (C-l) for all $l<k$, and let $L$ be a residual subspace of codimension $k$. By $W_{0}$-invariance it is equivalent to show (A-k), (B-k) and (C-k) for $L$ or for $w L$ so that we may and will assume that $R_{L}$ is a standard parabolic root subsystem. We apply Proposition 3.6 to $\tau$. Depending on the choices of the $\epsilon^{M}$ this defines a distribution $\mathfrak{X}_{L}$ supported on $L^{t}$. As was observed in [8, Proposition 3.10] the cycle $\xi_{L} \subset T_{L}$ (as in Proposition 3.6) depends only on the arrangement of poles and zeroes of $\eta$ which contain $L$, and we may take $\xi_{L}=\xi_{r_{L}}$, the cycle which is associated to the residual point $r_{L} \in T_{L}$ with respect to the affine Hecke algebra $\mathcal{H}\left(X_{L}, Y_{L}, R_{L}, R_{L}^{\vee}, F_{L}\right)$. In $T_{L}$ we are thus working with the rational form $\eta_{L}$ associated to the based root datum $\mathcal{R}_{L}=\left(X_{L}, Y_{L}, R_{L}, R_{L}^{\vee}, F_{L}\right)$, multiplicity function $q_{L}$, and base point $p_{L}=p T_{v}^{L} \cap T_{L, v} \in T_{L, v}$ deep in the negative chamber.

By the induction hypothesis $(\mathrm{B}-(\mathrm{k}-1))$ and $(\mathrm{C}-(\mathrm{k}-1))$ the collection of residual cosets of $\eta_{L}$ on $T_{L}$ satisfies the conditions of Lemma 4.3. By Lemma 4.3 there exist chambers of the (real projection of the) pole arrangement of $\eta_{L}$ such that for a base point $p_{L}^{\prime}$ in this chamber the residue contribution at $r_{L}$ with respect to $p_{L}^{\prime}$ and the form $\eta_{L}$ is nonzero and is a distribution of order $o_{L}$. By Proposition 2.8 there exist $w \in W_{L}$ such that $p_{L}^{\prime}=w^{-1}\left(p_{L}\right)$ is in one of the chambers so that the residue at $r_{L}$ is nonzero. By the $W_{L}$ quasi-invariance of $\eta_{L}$ this is equivalent to saying that the residue at $r_{w L}$ is nonzero with respect to the base point $p_{w L}=p T_{v}^{w L} \cap T_{w L, v}$ in $T_{w L}$. Hence by Proposition 3.6 and the text following this Proposition we see that $\mathfrak{X}_{w L}$ is nonzero. By the induction hypothesis $w L^{t}$ is not contained in a larger tempered residual coset. By Remark 3.7 the (possible) ambiguity of $\mathfrak{X}_{w L}$ (as a consequence of choices in the $\epsilon^{M}$ ) is limited to a subset of $w L^{t}$ of positive codimension. By Corollary 3.8(ii) this implies that $w L^{t}$ is in the support of $\mathfrak{X}$, proving $(\mathrm{A}-\mathrm{k})$.

By Corollary 5.8 we know that $\mathfrak{X}_{w L}$ is a measure. Hence Corollary 3.9 implies that $o_{w L}=0$. Since $o_{w L}$ is independent of $w$ (by $W_{0}$-invariance of $\eta$ ) and since $L$ is an arbitrary residual coset with codimension $k$ this proves $(\mathrm{B}-\mathrm{k})$.

Let us finally prove (C-k), arguing by contradiction. Since $o_{w L}=0$, the text after Proposition 3.6 and Corollary 3.8(ii) imply that $\mathfrak{X}_{w L}$ is the boundary value at $w L^{t}=r_{w L} T_{u}^{w L}$ of the rational function $\rho^{w L}\left(t^{w L}\right)=$ 


$$
\begin{aligned}
P^{w L}\left(r_{w L} t^{w L}\right) / Q^{w L}\left(r_{w L} t^{w L}\right) & \text { with } \\
P^{w L}(t) & =\Delta^{L}\left(w^{-1} t\right) \Delta^{L}\left(w^{-1}\left(t^{-1}\right)\right) \\
Q^{w L}(t) & =D^{L}\left(w^{-1} t\right) D^{L}\left(w^{-1}\left(t^{-1}\right)\right) .
\end{aligned}
$$

By the form of the factors of $\rho^{w L}$ the intersection of a pole or a zero of $\rho^{w L}$ is either empty or of real codimension 1 in $w L^{t}$. Assume that there exists a residual coset $M \neq L$ such that $M^{t} \subset L^{t}$. Since $o_{L}=0$ this implies that $i_{M}=o_{M}+\operatorname{codim}(M)>o_{L}+\operatorname{codim}(L)=i_{L}$. Hence in this situation there exists a codimension 1 coset in $w L^{t}$ along which $\rho^{w L}$ has a pole of order at least 1 .

Let $w L^{t, r e g} \subset w c T_{u}$ be the locally closed subset which is the complement in $w L^{t}$ of the singularities of $\rho^{w L}$ on $w L^{t}$. Recall that $\sum_{c \in \mathcal{C}} \mathfrak{X}_{c}$ is a probability Radon measure by Corollary 5.8. In particular, for all Borel measurable subsets $U \subset c T_{u}$ such that $\bar{U} \subset w L^{t, r e g}$ we have

$$
0 \leq \mathfrak{X}_{w c}\left(\chi_{U}\right) \leq 1
$$

On the other hand, outside the locus of singularities of $\rho^{w L},\left.\mathfrak{X}_{w c}\right|_{U}$ is, up to a nonzero constant $\mu$, equal to the boundary limit value (coming from some appropriate wedge which is immaterial at this point) of $\rho^{w L}$ on $U \subset w L^{t}$, i.e. integration of test functions supported on $U$ against $\omega^{w L}$. Thus for any open set $U \subset w L^{r e g, t}$ such that $\bar{U} \subset w L^{t, r e g}$ we have

$$
\mathfrak{X}_{w c}\left(\chi_{U}\right)=\mu \int_{U} \rho^{w L}(t) d^{w L}(t)
$$

Together with (6.1) this implies that $\mu \rho^{w L}$ is a positive integrable function on $w L^{r e g, t}$ with respect to Haar measure. But this contradicts the presence of a pole of $\rho^{w L}$ along a codimension 1 coset in $w L^{t}$. Hence our assumption was false, proving $(\mathrm{C}-\mathrm{k})$. This finishes the induction step, and we are done.

Corollary 6.2. For all $c \in \mathcal{C}, \mathfrak{X}_{c}$ is a sum over the $L \in \mathcal{L}$ such that $c_{L}=c$ of the push forward to $c T_{u}$ of a smooth measure on $L^{t}$. In view of Remark 3.7 the splitting if $\mathfrak{X}_{c}$ as sum of $\mathfrak{X}_{L}$ (for $L \in \mathcal{L}$ such that $\left.c_{L}=c\right)$ such that each $\mathfrak{X}_{L}$ is a smooth measure on $L^{t}$ is unique. We can take all $\epsilon^{L}$ in Proposition 3.6 equal to 1.

\section{Implications for root systems}

In this section we apply the previous results to prove the Theorems 1.1. 1.2 and 1.3 announced in the introduction. 
Theorem 7.1. For any coset $L \subset T$ we have $o_{L} \leq 0$. Explicitly this means that for all cosets $L \subset T$ :

$$
\left|\left\{\alpha \in R_{0}|\alpha|_{L}=q_{\alpha^{\vee}}\right\}\right|-\left|\left\{\alpha \in R_{0}|\alpha|_{L}=1\right\}\right| \leq \operatorname{codim}(L)
$$

In particular a coset $L$ is $\eta$-residual in the sense of Definition 3.2 if and only if $o_{L}=0$.

Proof. The first assertion is Theorem 6.1(B). The second assertion follows from this. Indeed, let $o_{L}=0$ and let $M \supset L$ be the connected component containing $L$ of the intersection of pole hypersurfaces $L_{m} \subset T$ of $\eta$ which contain $L$. Then $i_{M} \geq i_{L}$ and thus, since $o_{L}=0$ and $o_{M} \leq 0$ we have $\operatorname{codim}(M) \leq \operatorname{codim}(L)=i_{L} \leq i_{M} \leq i_{M}-o_{M}=\operatorname{codim}(M)$. It follows that $L=M$, and that $L$ is residual in the sense of Definition 3.2 .

Proof of Theorem 1.1. The statement that a residual point $v$ satisfies $o(v)=0$ is the special case of Theorem 7.1 where $L$ is a point in $T_{v}$, rephrased additively (using the exponential isomorphism between $V=\operatorname{Lie}\left(T_{v}\right)$ and the vector group $T_{v}$, with $\left.k_{\alpha}=\log \left(q_{\alpha^{\vee}}\right)\right)$. Let us prove that there are finitely many residual points. By Lemma 4.1 every residual point $v$ lies on at least one residual line $L$. Since $o_{L}=0$ we have for a generic point $w \in L$ that $o(w)=-1$. Hence every residual line $L$ contains at most finitely many residual points. A residual line $L$ is uniquely determined by a pair $\left(R_{L}, r_{L}\right)$ where $r_{L}$ is the unique residual point of $R_{L}$ such that $L=r_{L}+V^{L}$. Hence by induction on the rank the number of residual lines $L$ is finite. We conclude that the set of residual points for $R_{0}$ is finite.

The following corollary shows that the notion of residual cosets which we have used here in this note coincides with the original recurrent definition proposed in [5] and [8]:

Corollary 7.2. We can alternatively define residual cosets recurrently as follows. $T$ is a residual coset, and in general a coset $L \varsubsetneqq T$ is residual if and only if there exists a residual coset $M \supset L$ such that $\operatorname{codim}(L)=\operatorname{codim}(M)+1$ and such that $i_{L} \geq i_{M}+1$ (in fact it is clear by Theorem $6.1(B)$ that only the case $i_{L}=i_{M}+1$ will occur).

Proof. By Theorem 7.1 this is a consequence of [8, Lemma A.11].

Remark 7.3 (Theorem 1.1 and Richardson's Dense Orbit Theorem). In the special case of equal parameters $k_{\alpha}=k$ there is an alternative conceptual proof of Theorem 1.1. Without loss of generality we assume that $k_{\alpha}=k=2$ for all $\alpha \in R_{0}$. Given $v \in V$ we denote by $R_{v} \subset R_{0}$ 
the root subsystem $R_{v}=\left\{\alpha \in R_{0} \mid \alpha(v) \in 2 \mathbb{Z}\right\}$ and we let $V_{v}$ be the span of $R_{v}^{\vee}$. Observe that $o\left(R_{0}, V, 2 ; v\right) \leq o\left(R_{v}, V_{v}, 2 ; v\right)$. We choose positive roots $R_{v,+} \subset R_{v}$ such that $v$ is dominant integral for $R_{v}$. Let $G$ be the semisimple complex group of adjoint type with root system $R_{v}$, and let $P \subset G$ be the parabolic subgroup of $G$ whose Lie algebra is $\mathfrak{p}=$ $V_{v, \mathbb{C}}+\sum_{\alpha: \alpha(v) \geq 0} \mathfrak{g}_{\alpha}$. Let $P=L U$ be the Levi decomposition of $P$. By [2, Proposition 5.8.1] we see that $o\left(R_{v}, U_{v} ; v\right)=\operatorname{dim}\left(U / U^{\prime}\right)-\operatorname{dim}(L)$. By Richardson's Dense Orbit Theorem [2, Theorem 5.2.1] (also see [2, Proposition 5.8.2]) applied to $P$ we see that $o\left(R_{v}, U_{v} ; v\right) \leq 0$, proving Theorem 1.1 in this special case.

Theorem 7.4. Let $r \in T$ be a residual point with polar decomposition $r=c s \in T_{v} T_{u}$. Let $r^{*}=c^{-1} s$. Then $r^{*} \in W\left(R_{0, s}\right) r$, where $R_{0, s} \subset R_{0}$ is the root subsystem $\left\{\alpha \in R_{0} \mid \alpha(s)=1\right\}$.

Proof. The real point $c \in T_{v}$ is a residual point for the root datum $\left(R_{0, s}, X, R_{0, s}^{\vee}, Y\right)$. Indeed, it follows straight from the definition that $o_{\{c\}}\left(R_{0, s}\right)=o_{\{r\}}\left(R_{0}\right)$. Hence it suffices to prove the case $s=1$. By Theorem 6.1 we know that $W_{0} r$ is in the support of $\nu$. Now apply Proposition 5.1 .

Proof of Theorem 1.2. Theorem 1.2 is the special case where $r \in T_{v}$ is a real residual point in $T$ (rephrased additively).

Proof of Theorem 1.3. Suppose that Theorem 1.3 does not hold. Let $L=v_{L}+V^{L} \subset V=\operatorname{Lie}\left(T_{v}\right)$ be a residual affine subspace of positive dimension whose center $v_{L}$ is a residual point of $V$. Then $\left\{\exp \left(v_{L}\right)\right\} \in$ $T_{v}$ is a residual point (where we take $q_{\alpha} \vee=\exp \left(k_{\alpha}\right)$ ), and $\exp v_{L}+i V^{L}$ is the tempered part of a residual coset in $T$ properly containing the residual point $\exp v_{L}$. This contradicts Theorem 6.1(C).

Remark 7.5 (Theorem 1.3 and the Bala-Carter Theorem). Assume we are in the situation of Remark 7.3. Let $G$ be the complex semisimple group of adjoint type with root system $R_{0}$. By the Bala-Carter Theorem [2, Theorem 5.9.5] the unipotent orbits of $G$ are parameterized by $G$ conjugacy classes of pairs $(M, P)$ with $M \subset G$ a Levi subgroup and $P \subset M^{\prime}$ a distinguished parabolic subgroup. Using [8, Proposition B1] it is easy to see that a center $r_{L}$ of a residual affine subspace $L=$ $r_{L}+V^{L}$ which is in addition dominant is the weighted Dynkin diagram of the unipotent orbit $C_{(M, P)}$ with $M=M\left(R_{L}\right)$ and $P=P\left(r_{L}\right) \subset M$ the unique distinguished parabolic subgroup corresponding to the $R_{L^{-}}$ residual point $r_{L}$. By [2, Proposition 5.6.8] we see that if $r_{L}, r_{L_{1}}$ are dominant and are the respective centers of two residual affine subspaces $L, L_{1}$, then $r_{L}=r_{L_{1}}$ implies that $L_{1}=w L$ for some $w \in W_{0}$. 


\section{Temperedness of local traces $\chi_{t}$}

From general principles of harmonic analysis (see [1]) it should be expected that the support of the Plancherel measure $\mathcal{H}$ consists of the set of irreducible tempered representations. We show here that this indeed follows from the residue method and the basic technique of taking the weak constant part of a tempered representation along a standard parabolic subquotient algebra (see [3]). In addition we will find that the central support of the set of discrete series representations and the central support of the set of summands of properly induced tempered representations are disjoint.

The results of this section can be found in [8] but the proofs in [8] are inaccurate at some points (based on an earlier inaccuracy that occurred in [4]). We use the opportunity to correct the arguments here.

First of all we remark that the definition [8, Definition 3.9] of the dual central configuration $\mathcal{L}_{L}$ in $T_{L}$ is not correct. The correct definition is that $\mathcal{L}_{L}$ consists of all the $\operatorname{cosets} c_{L} T_{M, v}$ with $M$ in the intersection lattice generated by the central arrangement $\mathcal{L}^{L}$ in $T_{v}$ (and not just the $M \in \mathcal{L}^{L}$ which are coming from a residual coset as was written erroneously in [8]). With this understood [8, Proposition 3.12] is correct as stated.

Theorem 8.1. For all $t \in S$ the local traces $h \rightarrow \chi_{t}(h)$ are tempered.

Proof. The temperedness of the local traces is equivalent, by Casselman's criterion, to the statement that the real projection to $T_{v}$ of the support of the nonsymmetric measure $\mathfrak{X}$ (which is associated to $\left.\tau\right|_{\mathcal{A}}$ via the residue lemma) is contained in the closure of the anti-dual cone $T_{v}^{-}$of the positive Weyl chamber. This is a basic feature of the residue computations. Namely if a residual coset $w L$ is such that its center $w c_{L}$ is not in the closure of $T_{v}^{-}$then $\chi_{w L}=0$ by [8, Proposition 3.12].

The next result gives an alternative for the proof of [8, Lemma 3.31]. We remark that the proof as in [8], directly using [8, Proposition 3.12], can be corrected too, but this becomes somewhat more complicated by the weakening of [8, Proposition 3.12] mentioned above.

Corollary 8.2. Let $S^{d}$ denote the union of the $W_{0}$-orbits of residual points, and $S^{c}$ the union of the $W_{0}$-orbits of the tempered forms of the residual cosets of positive dimension. Then $S=S^{d} \sqcup S^{c}, S^{d}$ is the central support of the set of discrete series representations of $\mathcal{H}$ and $S^{c}$ is the central support of the set of irreducible tempered characters which are not in the discrete series. 
Proof. We have $S=S^{d} \sqcup S^{c}$ by Theorem 6.1(C). Suppose by induction on the rank of $X$ that the statement is true for all proper standard parabolic subquotient algebras $\mathcal{H}\left(X_{P}, Y_{P}, R_{P}, R_{P}^{\vee}\right)$. By the theory of the weak constant term (see [3]) it follows that if an irreducible tempered representation $\pi$ is not a discrete series representation then $\pi$ is a summand of a tempered standard module which is induced from a discrete series representation of a proper standard parabolic subquotient algebra $\mathcal{H}\left(X_{P}, Y_{P}, R_{P}, R_{P}^{\vee}\right)$. By the induction hypothesis and the fact that $S^{d} \cap S^{c}=\emptyset$ the central character of $\pi$ will belong to $S^{c}$. Thus the tempered irreducible characters supported by $S^{d}$ are discrete series characters.

It is known that an irreducible representation $\pi$ of $\mathcal{H}$ is a discrete series iff the mass of $\pi$ in the Plancherel measure is positive (see [8, Theorem 2.25]). Hence by the absolute continuity of the measure $\nu$ (Corollary 6.2) and the fact that $\nu$ is the push-forward of the Plancherel measure (see Corollary 5.7) to $\operatorname{MaxSpec}(\mathcal{Z})$ (equipped with the analytic topology) there can be no discrete series supported by $S^{c}$. Therefore the above and Theorem 6.1 imply that the central support of the discrete series representations is equal to $S^{d}$.

\section{References}

[1] J.N. Bernstein, On the support of Plancherel measure, J. Geom. Phys. 5 (1988), no. 4, 663-710.

[2] R.W. Carter, Finite groups of Lie type Wiley Classics Library, John Wiley and sons, Chichester, UK.

[3] P. Delorme, E.M. Opdam, The Schwartz algebra of an affine Hecke algebra, math.RT/0312517.

[4] G.J. Heckman, E.M. Opdam, Yang's system of particles and Hecke algebras, Annals of mathematics 145 (1997), pp. 139-173.

[5] G.J. Heckman, E.M. Opdam, Harmonic analysis for affine Hecke algebras, Current Developments in Mathematics (S.-T. Yau, editor), 1996, Intern. Press, Boston.

[6] J.N. Mather, Differentiable Invariants. Topology 16 (1977), 145-155.

[7] E.M. Opdam, A generating formula for the trace of the Iwahori-Hecke algebra, Progress in Mathematics 210 (2003), pp. 301-323 (also math.RT/0101006).

[8] E.M. Opdam, On the spectral decomposition of affine Hecke algebras, Journal of the Inst. of Math. Jussieu 3(4), 531-648 (2004).

[9] V. Poénaru, Singularités $C^{\infty}$ en Présence de Symétrie. LNM 510, SpringerVerlag, Berlin, 1976.

Korteweg de VRies Institute for Mathematics, University of Amsterdam, Plantage Muidergracht 24, 1018TV Amsterdam, The NetherLANDS, EMAIL: OPDAM@SCIENCE.UVA.NL 